\title{
Monitoring safety and effectiveness of Tafamidis in transthyretin amyloidosis in Italy: a 3-year longitudinal multicenter study in a non-endemic area
}

\author{
Andrea Cortese ${ }^{1 *}$, Giuseppe Vita ${ }^{2}$, Laura Obici ${ }^{3}$, Marco Sabatelli ${ }^{4}$, Gian Maria Fabrizi ${ }^{5}$, Angelo Schenone ${ }^{6}$, \\ Giampaolo Merlini ${ }^{3}$, Davide Pareyson ${ }^{7}$
}

From First European Congress on Hereditary ATTR amyloidosis

Paris, France. 2-3 November 2015

\section{Background}

Tafamidis is a transthyretin (TTR) stabilizer able to prevent mutated TTR tetramer dissociation into amyloidogenic monomers. There have been a few encouraging studies on safety and long-term efficacy of Tafamidis in early-onset Val30Met TTR-familial amyloid polyneuropathy (TTR-FAP) patients. However, less is known about its efficacy in later stages of the disease and in non-Val30Met mutations.

\section{Methods}

Multi-center observational study on symptomatic TTRFAP patients prescribed to receive tafamidis. We followed up patients according to a standardized protocol including general medical, cardiological and neurological assessments at baseline and every 6 months up to 3 years.

\section{Results}

61 (42 males) patients were recruited. Only $28 \%$ of enrolled subjects had the common Val30Met mutation, mean age of onset was remarkably late (59 years) and $18 \%$ was in an advanced disease stage at study entry. Tafamidis proved safe and well-tolerated. One third of patients did not show significant progression along 36 months, independently from mutation type and disease stage. Neurological function worsened particularly in the first 6 months but slowed significantly thereafter. Fifteen percent of patients showed cardiac disease progression and $30 \%$ new onset of cardiomyopathy. A higher $\mathrm{mBMI}$ at baseline was associated with better preservation on neurological function.

${ }^{1}$ C. Mondino National Neurological Institute, General Neurology, 27100, Pavia Italy

Full list of author information is available at the end of the article

\section{Conclusions}

Neuropathy and cardiomyopathy progressed in a significant proportion of patients despite treatment. However, the worsening of neurological function slowed after the first 6 months and also subjects with more advanced neuropathy, as well as patients with non-Val30Met mutation, benefited from Tafamidis treatment. Body weight preservation is an important favorable prognostic factor.

\section{Authors' details \\ ${ }^{1}$ C. Mondino National Neurological Institute, General Neurology, 27100, Pavia, Italy. ${ }^{2}$ University of Messina and NEMO SUD Center for Neuromuscular Disorders, Fondazione Aurora Onlus, Department of Neurosciences, 98121, Messina, Italy. ${ }^{3}$ Amyloidosis Research and Treatment Center, Department of Molecular Medicine, Fondazione Istituto Di Ricovero e Cura a Carattere Scientifico Policlinico San Matteo and University of Pavia, 27100, Pavia, Italy. ${ }^{4}$ Institute of Neurology, Catholic University of Sacred Heart, Department of Geriatrics, Neurosciences and Orthopedics, 00118, Roma, Italy. ${ }^{5}$ University of Verona, Department of Neurological, Neuropsychological, Morphological and Motor Sciences, 37010, Verona, Italy. ' University Federico II of Naples, Department of Neurosciences, Reproductive and Odontostomatological Sciences, 80121, Napoli, Italy. 'IRCCS Foundation, C. Besta Neurological Institute, Department of Neurolog, 20124, Milano, Italy.}

Published: 2 November 2015

doi:10.1186/1750-1172-10-S1-P6

Cite this article as: Cortese et al:: Monitoring safety and effectiveness of Tafamidis in transthyretin amyloidosis in Italy: a 3-year longitudinal multicenter study in a non-endemic area. Orphanet Journal of Rare Diseases 2015 10(Suppl 1):P6. 